\title{
Effect of Hippuric Acid on the Growth, Structural and Optical Properties of Non Linear Optical Crystals
}

\author{
A Kumaresh \\ GRD Centre for \\ Materials Research, \\ PSG College of \\ Technology, \\ Coimbatore, India
}

\author{
R. Arun Kumar \\ GRD Centre for \\ Materials Research, \\ PSG College of \\ Technology, \\ Coimbatore, India
}

\author{
G. Sankar \\ GRD Centre for \\ Materials Research, \\ PSG College of \\ Technology, \\ Coimbatore, India
}

\author{
P. Selvakumar \\ GRD Centre for \\ Materials Research, \\ PSG College of \\ Technology, \\ Coimbatore, India
}

\footnotetext{
M. Arivanandhan,

Shizuoka University,

Hamamatsu, Japan

rmarivu@ipc.shizuoka.

ac.jp
}

\author{
Y. Hayakawa, \\ Shizuoka University, \\ Hamamatsu, Japan, \\ royhaya@ipc.shizuoka.
}

\author{
H. Kawai, \\ Shizuoka University, \\ Hamamatsu, Japan
}

\begin{abstract}
Non-linear optical potassium dihydrogen phosphate (KDP) and ammonium dihydrogen phosphate (ADP) single crystals with $1 \mathrm{~mol} \%$ of hippuric acid dopant were grown from aqueous solution by employing slow evaporation technique. With appropriate dopants, the KDP and ADP crystals exhibit several desirable properties. Crystallinity of the grown crystals was studied by powder XRD analysis. The bonding structure and molecular associations were analyzed by FTIR spectroscopy. It also confirms the functional groups present in the grown crystals. UV-Vis-NIR spectral analysis was carried out to study the optical characteristics of the crystals which reveal that the cutoff wavelength for both hippuric acid doped KDP and ADP crystals is around $300 \mathrm{~nm}$. Second harmonic generation (SHG) test adopting the Kurtz Perry technique revealed that the SHG efficiency has increased due to doping when compared with pure KDP and ADP crystals.
\end{abstract}

Keywords: solution growth, single crystals, Powder XRD, UV-Vis-NIR, FTIR, Powder SHG test, optical property.

\section{INTRODUCTION}

Potassium dihydrogen phosphate (KDP) and ammonium dihydrogen phosphate (ADP) are promising materials that play an important role in piezoelectric, ferroelectric, electrooptic and nonlinear optical industries. This material attracts the many researchers probably because of their simple structure and many desirable properties associated with a hydrogen bond system involving a large isotopic effect, broad transparency range, high laser damage threshold and relatively low production cost $[1,2]$.

Nonlinear optics (NLO) has wide applications in the field of telecommunication and information devices [3-7]. Nonlinear optics gives much importance in photonics and optoelectronics technologies. New nonlinear optical frequency conversion materials have a significant impact on laser technology, optical communication and optical data storage [8-10].Recent interest in quantum electronics has focused on finding new materials for efficient second harmonic electro optic modulation [11].

The organic doping material contains an optically active atom i.e. it will have hyper polar molecule, which favors the formation of big size crystals [12]. Hippuric acid (HA) with molecular formula $\mathrm{C}_{6} \mathrm{H}_{5}-\mathrm{CO}-\mathrm{NH}-\mathrm{CH}_{2}-\mathrm{COOH}$ is also referred as benzamino acetic acid is an organic nonlinear material. The molecular and chemical structure of HA are shown in the Figure 1 (a) and Figure 1 (b) respectively. It is formed in the order of orthorhombic structure. It is an excellent material for second harmonic generation because of its high conversion efficiency [13].

The organic NLO materials are sufficiently good over the inorganic materials because they have high nonlinear response. Organic materials are often formed by weak Van der waals force and hydrogen bonds and hence possess high degree of delocalization. The amino acids are the famous organic materials which play a vital role in the field of nonlinear optical crystal growth. Many members of natural 
amino acids are individually exhibiting the nonlinear optical properties because they have a donor $\mathrm{NH}_{2}$ and acceptor $\mathrm{COOH}$ group and the intermolecular charge transfer is also possible [14]. Since hippuric acid exhibits good nonlinear optical properties, it is of interest to dope it in KDP and ADP crystals. In this work we have investigated the effect of organic dopant in Potassium dihydrogen phosphate (KDP) and ammonium dihydrogen phosphate (ADP) in order to increase the NLO efficiency.

\section{SINGLE CRYSTAL GROWTH}

Single crystals of 1 mole\% hippuric acid doped KDP and ADP crystals were grown by solution growth employing slow evaporation technique. The starting materials were subjected to repeated recrystallization to improve their quality. HA salt was added to the powdered crystalline samples of KDP and ADP in 1 mole\% separately to form saturated solutions. The solutions were thoroughly stirred for homogenization and then filtered into a borosil beaker using Whatmann filter paper. The beakers containing the solutions were closed with perforated cover and kept in a dust free atmosphere. Transparent colorless single crystals with the dimensions 35 $\mathrm{mm} \times 8 \mathrm{~mm}$ x $4 \mathrm{~mm}$ for HAKDP and $54 \mathrm{~mm}$ x $20 \mathrm{~mm}$ x 11 $\mathrm{mm}$ for HAADP were harvested within 3-4 weeks. The photographs of HAKDP and HAADP crystals are shown in Figure 1 (a), (b)

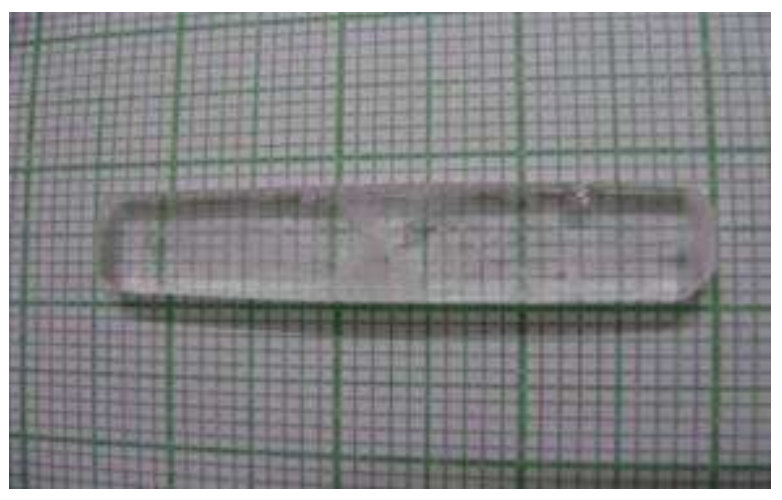

Figure 1 (a) Single crystal of HA doped KDP

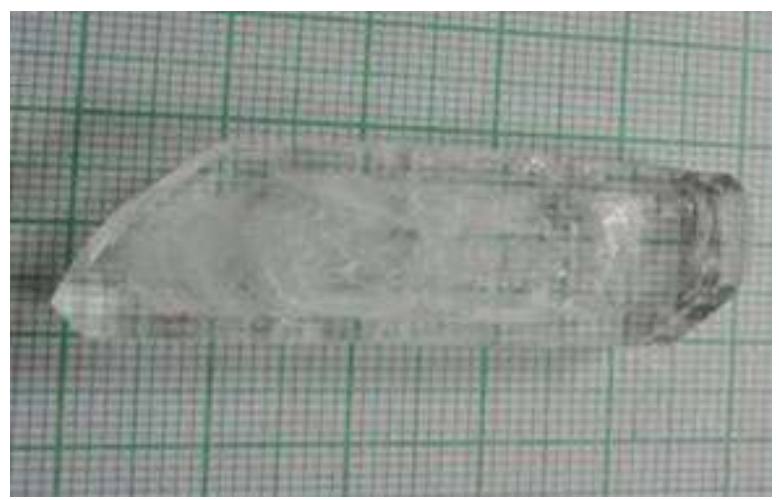

Figure 1 (b) Single crystal of HA doped ADP

\section{RESULT AND DISCUSSION}

\subsection{Powder XRD analysis}

Powder X-ray diffraction analysis was performed to confirm the quality of the grown crystals and to identify the cell dimensions using Rigaku X-ray diffractometer with $\mathrm{CuK}_{\alpha}$ radiations $(\lambda=1.540 \AA)$ within the $2 \theta$ range $20^{\circ}-75^{\circ}$. The powder XRD pattern of HAADP and HAKDP crystals are shown in Figure 2 (a) and 2 (b). Using XRDA the lattice parameter values of grown crystals were calculated as $a, b=$ $7.4993 \AA, c=7.5525 \AA$ for HAADP single crystals and it belongs to tetragonal crystal structure. For HAKDP $\mathrm{a}=10.5058 \AA, \mathrm{b}=10.4495 \AA, \mathrm{c}=6.9186 \AA$ and the crystal system belongs to Orthorhombic. The incorporation of impurity (hippuric acid) in the crystals gives a slight variation in the lattice parameters and the cell volume for the doped crystal naturally varies. It is evident for the incorporation of the dopant, unit cell volume gets increased.

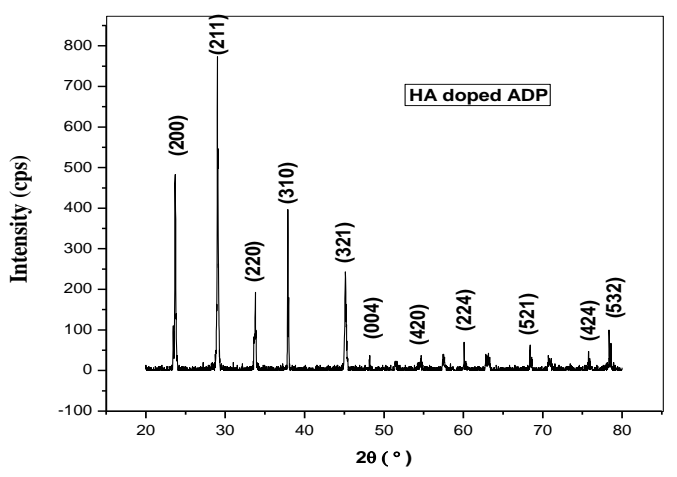

Figure 2 (a) Powder XRD spectrum of HAADP crystal

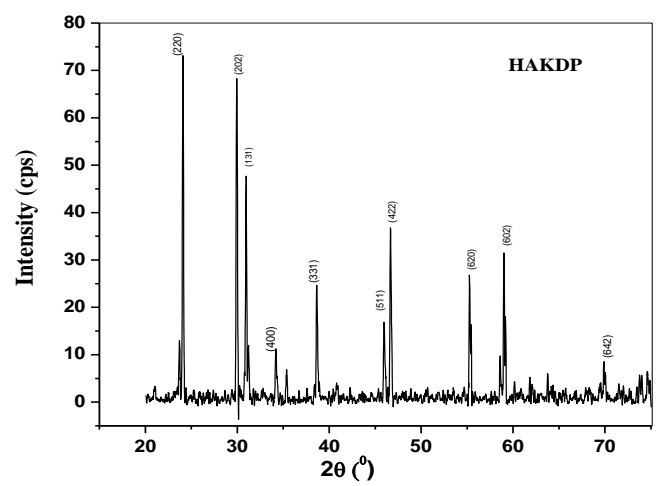

Figure 2 (b) Powder XRD spectrum of HAKDP crystal

\subsection{UV-VIS-NIR optical analysis}

The optical properties of the material are important, as they provide information on the electronic band structure, localized state and the type of optical transitions because the absorption of UV and visible light involves promotion of the electron in $\sigma$ and $\pi$ orbital from the ground state to higher energy states [15]. The UV-Vis- NIR absorption and transmittance 
spectrum was recorded using Jasco V-570 UV-Vis-NIR spectrophotometer. From both the spectrum figure 3 (a), (b), it is found that the cut off wavelength of the hippuric acid doped $\mathrm{KDP}$ and ADP crystals is around $300 \mathrm{~nm}$. It is found that there is an absence of absorption in the region between 340 $\mathrm{nm}$ and $1200 \mathrm{~nm}$, which are the most desirable properties of the material for NLO application.

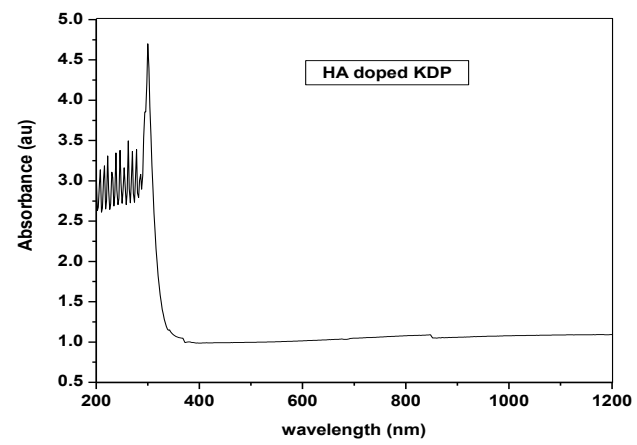

Figure 3 (a) Absorbance curve of HA doped KDP

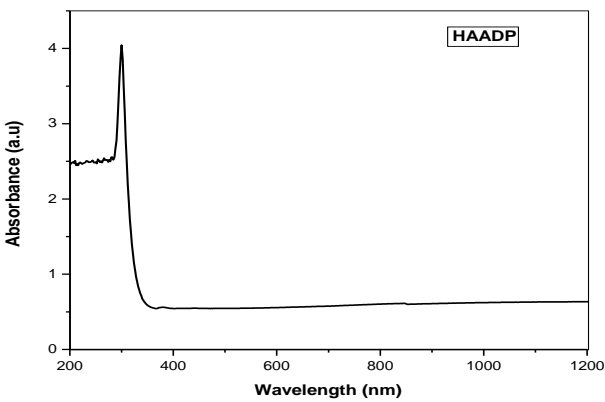

Figure 3 (b) Absorbance curve of HAKDP

\subsection{FTIR Analysis}

FTIR spectroscopy is effectively used to identify the functional groups in order to determine the molecular structure of the compounds. The FTIR spectrum was recorded by Shimadzu FTIR-8400S spectrometer technique in the range from $400 \mathrm{~cm}^{-1}$ to $4000 \mathrm{~cm}^{-1}$.

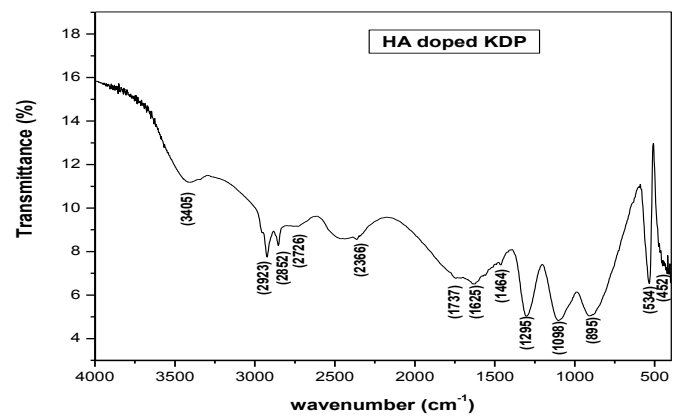

Figure 4 (a) FTIR spectrum of HAADP crystal
Figure 4 shows the FTIR spectrums of HAADP single crystal. FTIR spectra of both HAKDP and HAADP confirm the presence of functional groups in the grown crystals.

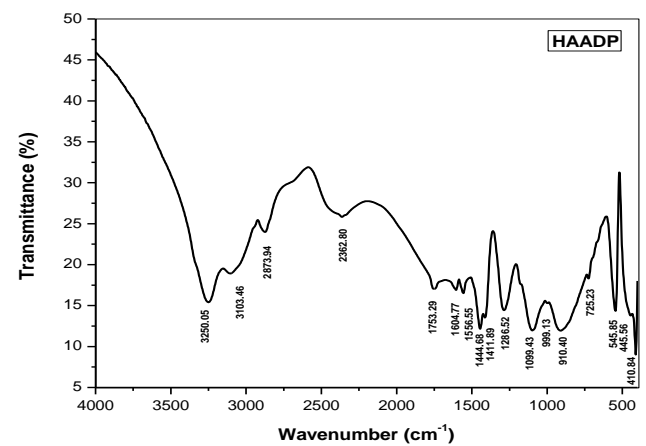

Figure 4(b) FTIR spectrum of HAADP crystal

\subsection{Powder SHG test}

Powder SHG test by the Kurtz-Perry technique was adopted for the initial testing of the grown crystals for frequency conversion applications. The fundamental beam of wavelength $1064 \mathrm{~nm}$ from a Q-switched Nd:YAG laser was used to test the second harmonic generation property of the grown HAADP and HAKDP crystals. Both the crystals were ground into fine powders and packed in micro tubes mounted in the path of laser pulses operating with a pulse width of $6 \mathrm{~ns}$ and a repetition rate $10 \mathrm{~Hz}$ and having an input energy of 0.68 $\mathrm{ms} /$ pulse. The second harmonic generation of the crystals was confirmed by a green emission with the of wavelength $532 \mathrm{~nm}$ from the samples. The second harmonic generation efficiency of $1 \mathrm{~mol} \%$ HA doped ADP and KDP crystals were found to be 1.5 and 1.2 times that of pure ADP and KDP crystals respectively.

\section{CONCLUSION}

Good quality of single crystals of hippuric (HA) acid doped KDP (HAKDP) and ADP (HAADP) were grown by solution growth technique for the first time. The structural characterization of the grown crystals was carried out by powder X-ray diffraction studies. UV-VIS-NIR optical analysis shows good optical transmittance in the entire visible region for the above mentioned crystal, also it is found that the UV cut off wavelength is around $300 \mathrm{~nm}$ and it can be used as a potential material for SHG in the visible region down to blue and violet lights, which makes it suitable for laser frequency doubling and related optoelectronic applications. The FTIR study confirms the presence of hippuric acid in the grown crystal 


\section{REFERENCES}

[1] Shirsat .M.D., Hussaini .S.S, Dhumane .N.R, and Dongre V.G. (2008).Crist.Res.Technol.43:756-761.

[2] Kannan .V., Bairava Ganesh R, Sathyalakshmi R., Rajesh N.P and Ramasamy .P, (2002). Mat. Lett. 56: 578.

[3] Chenthamarai .S,.Jayaraman D, Meera . Subramanian. C and Ramasamy.P, Mat. Chem. Phys. 64, 179 (2000).

[4] Chenthamarai S.,.Jayaraman D, Meera K., Santharagavan P., Subramanian C. Bocelli G. and.Ramasamy .P, Cryst.Engg.4,37 (2001).

[5] Rai R.N.,.Ramasamy P, and.Lan C.W. J.Cryst. Growth 235, 499 (2002).

[6] Vijayan N., Ramesh Babu R. Gunasekaran .M, Gopalakrishnan R., Kumaresan R .Ramasamy P and Lan C.W., J.Cryst.Growth 249, 309 (2003).

[7] Selvakumar S., Ravikumar S.M., Rajarajan K., Joseph A., Arul Pragasa, Rajasekaran S.A., Thamizharasan K., and Sagayaraj P. , J. Cryst.Growth 11, 2607 (2006).

[8] Angelimery P.A. and Dhanuskodi S., Cryst. Res. Technol. 36, 1231 (2001).
[9] Dhanuskodi S. and Vasantha K., Cryst. Res. Technol. 39, (2004). 259

[10] Ariponnammal S., Radhika S., Selva and R. VictorJeya N., Cryst. Res. Technol 40,786 (2005).

[11] HajaHameed A.S., Ravi G., Dhanasekaran R. and Ramasamy P. J. Cryst. Growth 212, 217 (2000).

[12] LakshmanaPerumal C.K., Archakkarvarthi A. and Santharagavan P. J. Cryst. Growth. 241, 200 (2002).

[13] Chemla D.S., Zyss J. (Eds), Nonlinear Optical Properties of Organic Molecules and Crystals, vol.1, Orlando, Academic Press, 1987, pp. 23-187.

[14] Krishna Mohan M., Jagannathan K., Ponnusamy S. and Muthamizhchelvan C., J. Phys. Chem. Solids.72 (2011) 1273-1278.

[15] Dhanaraj P.V., Rajesh N.P., Ramasamy P., Jayaprakasan, Mahadevan C.K., and Bhagavannarayana G., "Enhancement of stability of growth, structural and NLO properties of KDP crystals due to additive along with seed rotation," Crystal Research and Technology, vol.44, no.1,pp. 5460,2009 . 